Manuscript ID: PONE-D-19-25424R1 (revised)

S1_raw_images

The following images correspond to Fig3C of the manuscript

$\frac{\mathrm{ct}}{\mathrm{M} C} \frac{\mathrm{H}_{2} \mathrm{O}_{2}}{\mathrm{M} C \mathrm{C}} \frac{\mathrm{CNP} \text { 4h }}{\mathrm{M} C \mathrm{CNP} 24 \mathrm{~h}}$

\title{
sulfenic acids
}

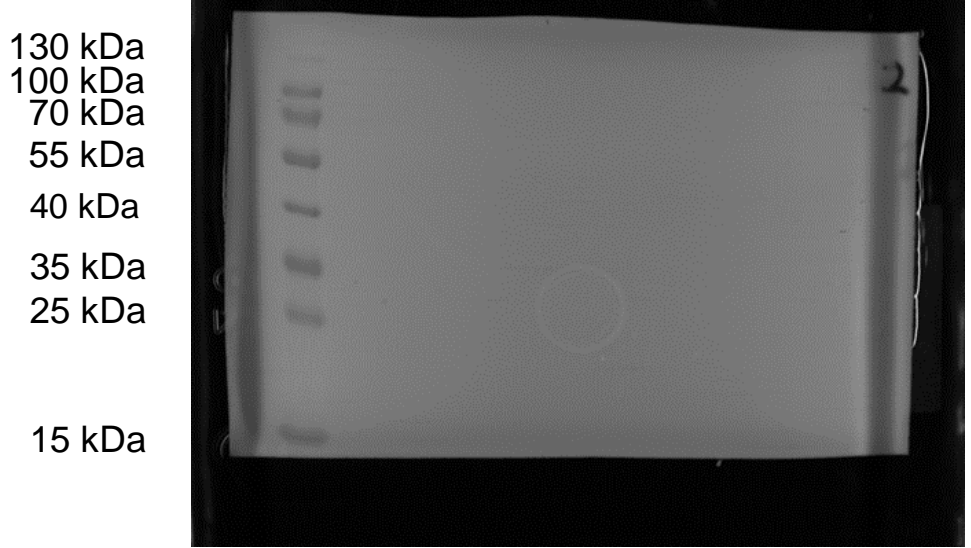

\section{Upper panel:}

A375 cells were separated into cytosolic (C) and mitochondrial (M) fraction after mock-treatment, incubation with $\mathrm{H}_{2} \mathrm{O}_{2}$ or incubation with CNP. Western Blot analysis was performed using an $\alpha$ hapten antibody raised against the oxidation product of sulfenic acid and dimedone. The antigenantibody complexes were visualized by an enhanced chemiluminescence system with the Fusion FX (Vilber Lourmat, Eberhardzell, Germany).

The image was taken at 8-14-18; exposure time was 3min 42sec.

\section{Lower panel:}

Corresponding PVDF membrane with markerMarker: PageRuler ${ }^{\text {TM }}$ Prestained Protein Ladder 10 to $180 \mathrm{kDa}$, Thermo Scientific 

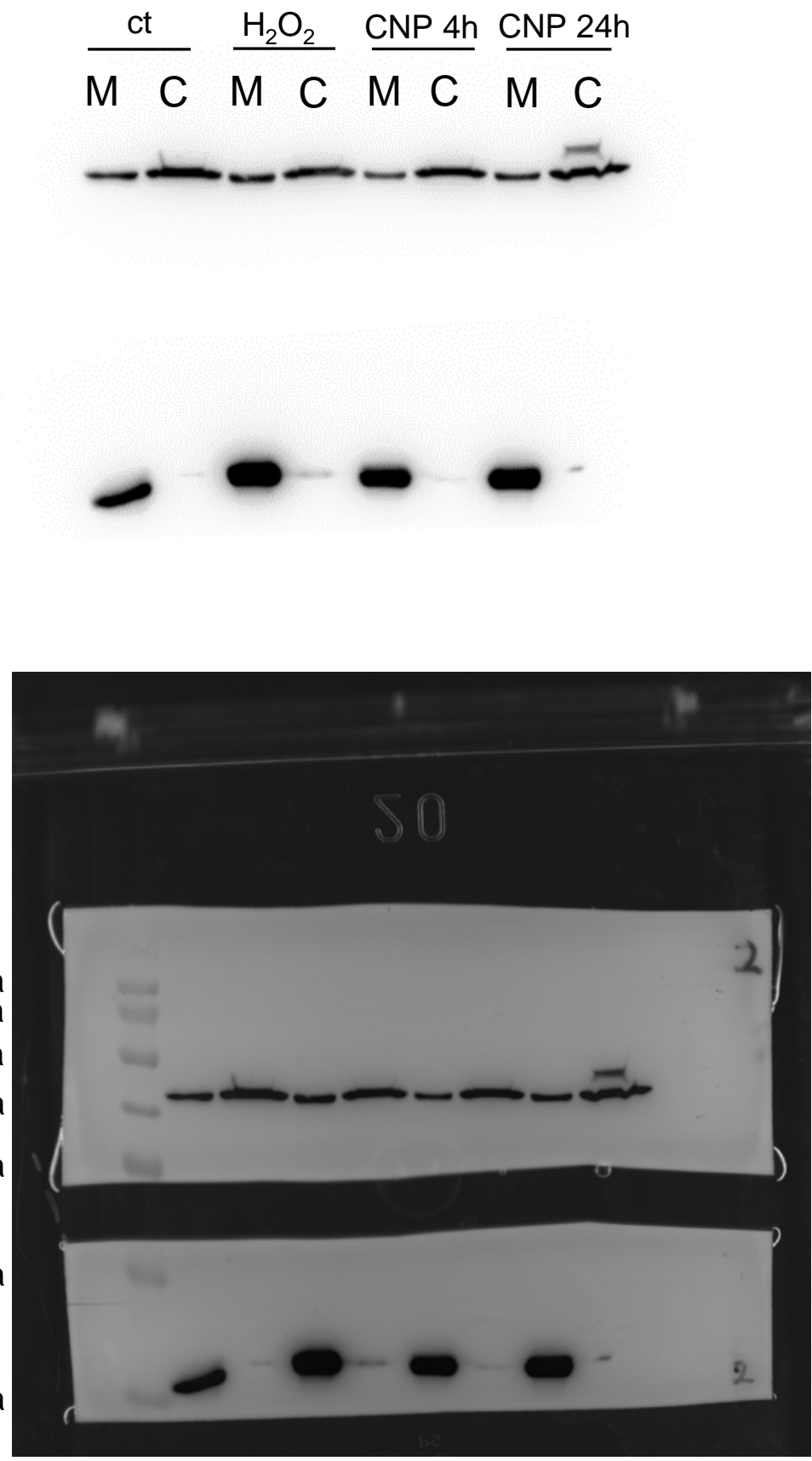

\section{Upper panel:}

A375 cells were separated into cytosolic (C) and mitochondrial (M) fraction after mocktreatment, incubation with $\mathrm{H}_{2} \mathrm{O}_{2}$ or incubation with CNP. Western Blot analysis was performed using anti-beta-actin and anti TOM-20 antibodies. Antigen-antibody complexes were visualized by an enhanced chemiluminescence system with the Fusion FX (Vilber Lourmat, Eberhardzell, Germany). The image was taken at 8-14-18, exposure time was $1 \mathrm{~min} 72 \mathrm{sec}$.

\section{Lower panel:}

Corresponding PVDF membranes with marker, merged with Western Blot Marker: PageRuler ${ }^{\text {TM}}$ Prestained Protein Ladder 10 to 180 kDa, Thermo Scientific 


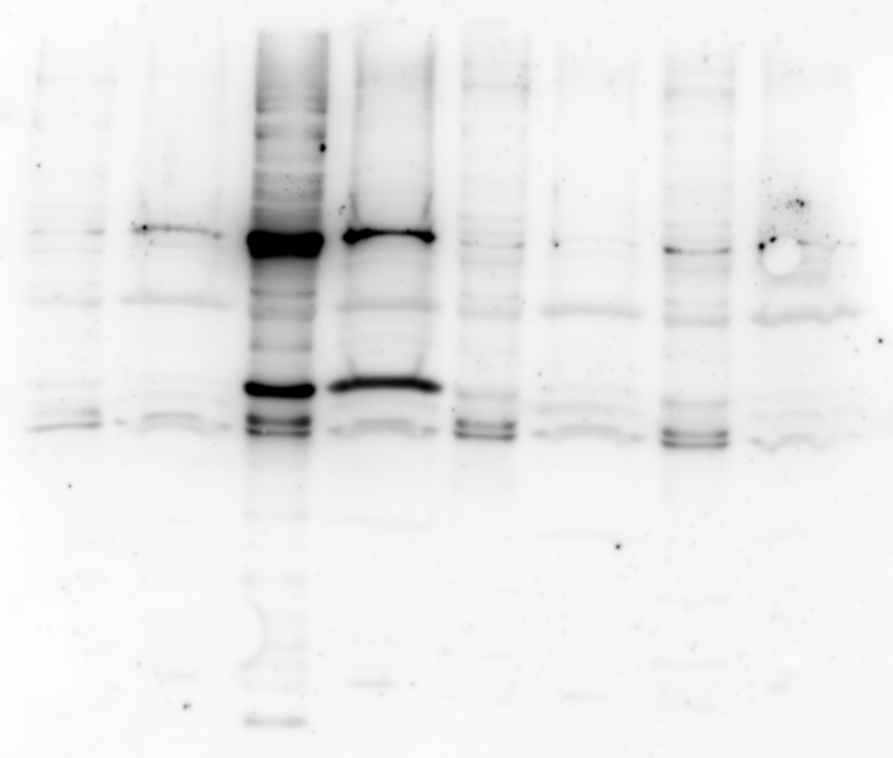


- - - - -
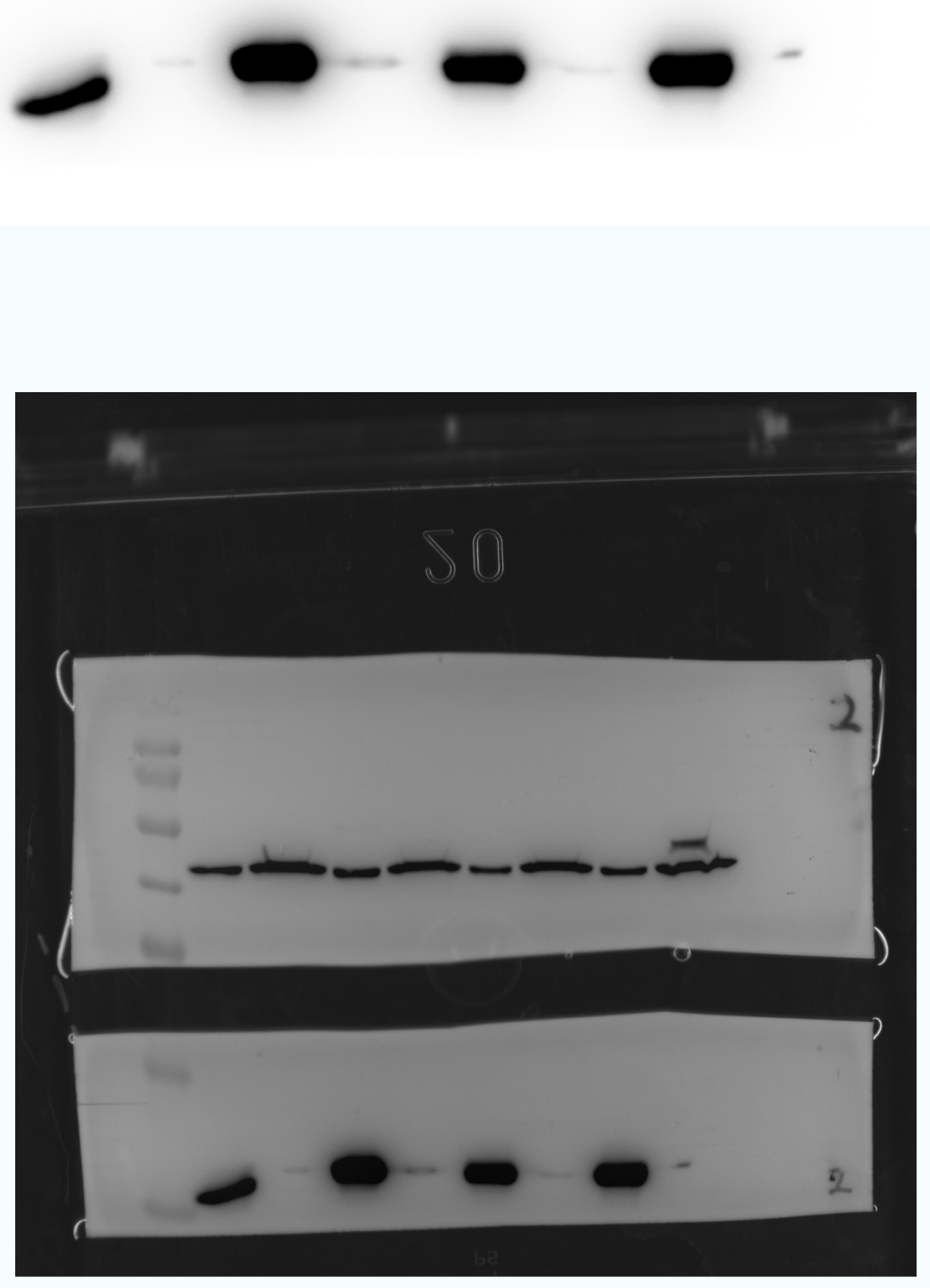